\title{
Home parenteral nutrition
}

\author{
J W L Puntis
}

The need for long term parenteral nutrition is no longer in itself adequate justification for keeping a child in hospital. With the development of both the nutritional support industry and hospital outreach services, parenteral nutrition at home has become a practical option. The necessary organisation and supervision remain complex, however, so that home parenteral nutrition (HPN) is best coordinated through a relatively small number of centres. Ideally, these should be able to provide a paediatric gastroenterology/nutrition service, involving a multidisciplinary nutritional care team which includes a paediatric surgeon.

\section{Home care}

Home care has been defined as 'the provision of equipment and services to the patient in the home for the purpose of restoring and maintaining his or her maximal level of comfort, function and health' ${ }^{1} \mathrm{HPN}$ is one of an increasing number of home care services available as alternatives to treatment in hospital. Despite recent evidence of an increase in home care for children, there is considerable scope for further expansion. ${ }^{2}$ For example, in the UK the growth of HPN has been much slower than in some other countries such as France and the USA. In North America, the main factors in the development of home care were pressure from insurers to reduce costs of treatment, the availability of equipment such as ambulatory infusion pumps, competition between private providers, and patient preference. ${ }^{3}$

The possible cost saving advantage of caring for patients at home holds obvious attractions for budget holders. For HPN, as well as other home care services however, there are few data available on cost effectiveness, ${ }^{4}$ and funding arrangements vary considerably from place to place. From the definition of home care given above ${ }^{1}$ it follows that there are major quality (and therefore cost) issues involved. With this in mind the British Association of Parenteral and Enteral Nutrition (BAPEN) has called for the establishment of local and national registers of patients in order to provide data on the numbers involved, the resource implications, and likely future trends. ${ }^{5}$ BAPEN has also recommended that expertise in HPN should be concentrated in a limited number of centres, perhaps with one adult and one paediatric unit per region. ${ }^{5}$
Among the most important considerations for paediatricians are that HPN offers children who would otherwise be institutionalised, the possibility of fully realising growth and developmental potential, a good quality of life, and a reduced risk of complications such as central venous catheter sepsis. ${ }^{67}$ In fact, most can attend school and take part in normal social life including sporting activities and holidays. 68

\section{Children who may need HPN}

Children with chronic intestinal failure are potential candidates for HPN. Initiating parenteral nutrition in the child with little or no prospect of eventually adapting to full enteral nutrition (such as one with a congenital enteropathy or after massive gut resection in the newborn period) still presents carers with a major ethical dilemma requiring careful consideration. ${ }^{9}$ The alternative to long term parenteral nutrition in some children is small bowel transplantation. Initial experience proved disappointing ${ }^{10}$ but technical advances now mean that surgery has progressed well beyond the experimental stage. Transplantation will probably be seen as a solution to failed (for whatever reason) $\mathrm{HPN}$, but at present is not readily available. Parenteral nutrition makes it possible to sustain long term growth and has greatly improved the prognosis of children with intestinal failure. In short bowel syndrome, for example, one recent study reported an increase in the survival of children with less than $40 \mathrm{~cm}$ of small intestine from $42 \%$ before 1980 to a figure now approaching $94 \% .{ }^{11}$ When there is $40-80 \mathrm{~cm}$ of small bowel survival in the region of $97 \%$ can be expected. ${ }^{11}$ Such results are indicative of a more aggressive approach to early nutritional support after massive loss of small bowel, with short bowel syndrome now comprising one of the most common diagnoses among children receiving HPN.

In a recent review of HPN in one UK centre, ${ }^{6}$ patients were evenly divided between those with short bowel, congenital enteropathy, and severe motility disorders (pseudoobstruction). Among a larger series of patients from France, short bowel was the most common condition, affecting 44 out of 112 children, motility disorders being the next largest group, followed by Crohn's disease, protracted diarrhoea, and immune deficiency. ${ }^{8}$ During an eight year period, 49 of these 
children graduated to full enteral nutrition, and 18 died. The present situation in the UK has been ascertained through a recent survey undertaken by the British Paediatric Surveillance Unit which identified 66 children on parenteral nutrition for longer than six months, 34 of whom were receiving HPN while eight were regarded as unsuitable.

Our own practice includes a regional neonatal medical and surgical service. We have considered HPN in children in whom the need for parenteral nutrition seemed likely to extend beyond six months. A number of other factors have a bearing on suitability for HPN. Severity of underlying disease must be taken into account as fluid losses and enteral tolerance have important implications, as does the presence of other medical conditions such as chronic lung disease. For example, if some enteral intake is tolerated, a separate infusion of lipid emulsion and the daily addition of vitamin preparations to the feed bag may be unnecessary. The overall clinical condition must be relatively stable.

Parents need to be highly motivated and want to take on the burden of responsibility for their child's day to day medical care. They must be able to cope not only with the emotional demands of HPN, but also master the technical aspects. Suitable housing and a supportive partner, relation, or friend who can share all aspects of care are both necessary prerequisites. In some conditions such as short bowel syndrome, specialised enteral support may also be needed ${ }^{12}$ and impose its own additional demands. ${ }^{13}$

\section{Nutritional nurse specialist}

A nurse specialist in nutritional support based on the paediatric unit is central to the success of HPN. The nurse has a crucial role in assessing the suitability of the family, providing structured teaching, organising the community support, providing the link back into hospital after discharge, and coordinating the input of all other professionals. Our nurse was funded from revenue supporting the existing nursing establishment, the appointment reflecting anticipation by our trust of an expansion in home care. The cost effectiveness of this change in nursing role was argued on the basis of estimates of the relative costs of HPN versus hospital care, and the benefits likely to accrue from the role of the nutrition nurse within a multidisciplinary nutritional care team ${ }^{14} 15$ providing nutritional support throughout the paediatric unit. ${ }^{16}$

\section{Assessment and teaching of parents}

Family assessment regarding suitability for HPN is undertaken informally by the nutritional nurse specialist in conjunction with the ward staff and primary health care team. This includes a home visit during which practical details such as the availability of storage space, power points, lighting, and hand washing facilities are reviewed. If there is agreement that the family is suitable for HPN and the home circumstances are adequate, detailed preparation begins. A teaching programme has to be formulated to make sure that the parents are confident and competent in all aspects of their child's care. This includes hand washing and aseptic technique, knowledge of central venous catheters, catheter care and dressing changes, care of the infusion pump, running feed solutions through and connecting to the central venous catheter, setting up the infusion and making the necessary connections, urine testing, temperature taking, problem solving, and what to do in an emergency.

The teaching programme is best undertaken during a period of several weeks when both parents are resident in hospital ${ }^{6}$ particularly if the family live a long distance from the HPN centre. In practice some parents are not able to be resident and teaching has been extended over a longer period of time. The teaching programme is individually tailored to meet their needs. Each session is timed during the day or early evening when convenient, and is limited to learning one particular skill, allowing time for practice. A written record of progress is kept, and only when competence is achieved in one area is a new subject introduced.

\section{Family held records}

Children on HPN may be seen by a large number of different health care professionals in a number of different locations (home, general practice, district hospital, HPN centre). Although parents have open access to the paediatric ward, we provide the family with a set of medical records both for their reference and for the benefit of any professionals they encounter who are unfamiliar with the child, such as new junior staff. A folder is compiled which includes an up to date medical summary, and a list of contact names and telephone numbers. The family and nutritional nurse draw up a care plan to fit in with the family's routine. The folder also includes written information to back up the teaching sessions in hospital, instruction manuals for equipment, problem solving advice, growth charts, and flow charts recording the results of biochemical monitoring results. Some of this information is readily available in the form of a well illustrated and parent friendly booklet. ${ }^{17}$

\section{Supply of equipment}

We have arranged this through one of the commercial home care companies and have used both Fastnet and Caremark. The nutritional nurse compiles a stock list of necessary equipment and disposables (see table 1) then places an order with the local representative of the home care company. The pharmacist liaises with the compounding unit with regard to the parenteral nutrition formulation, which by this time requires infrequent modification. We follow standard nutritional regimens ${ }^{18}$ modified to take into account enteral intake and fluid and electrolyte 
Table 1 Equipment needs at discharge in a HPN patient with short bowel

Chlorhexidine $1 / 200$ with alcohol $70 \%, 500 \mathrm{~m}$

Aquasept, $500 \mathrm{ml} ; 5 \mathrm{ml}$ dispenser

Manusept, $500 \mathrm{ml} ; 3 \mathrm{ml}$ dispense

Parenteral nutrition feed bags, one month supply

Refrigerator

IVAC 597 intravenous infusion pump

Folding trolley

IV stand

IVAC IV administration sets with $Y$ site and luer lock

IBEX-HP 0.22 micron high pressure vented filter set

Enteral feed (Chix, Maxijul, Calogen, Liquigen, Aminogram, pectin)

Kangaroo $500 \mathrm{ml}$ easy cap pump set (box of 36)

Kangaroo 2100 enteral feeding pump

Kangaroo 2100 enteral feed
Vygon nasogastric tubes

Vygon nasogastric
Blue litmus paper

Blue litmus pap
Stoma-hesive

Zinc oxide adhesive tape

NHS supplies wound care pack

Latex gloves ( 50 pairs)

Paper towels

5 ml Syringes

Hepflush 100 units heparin/ml, $2 \mathrm{ml}$ ampoules

Normal saline, $5 \mathrm{ml}$ plastic ampoules

Durapore tape

Mediwipes

Absorbent dressings

Gauze swabs

Urine Ketodiastix

Emla cream with Tegaderm dressings

Sterilising solution (Milton)

Disposal bin for 'sharps'

Yellow plastic waste bag

losses. Fluid bags and disposables are usually delivered each two weeks; adequate storage space at home is essential. The home care company provides a folding trolley, and a large refrigerator for two weeks' supply of parenteral nutrition feed bags. Disposal of sharps and clinical waste need special arrangements with the local authority. The council cleansing department is contacted so that yellow bags for clinical waste can be collected once a week for incineration (district nurses keep copies of the appropriate form). The electricity company is informed of the importance of maintaining supply.

\section{Costs and funding}

Bisset and colleagues estimated the annual cost of HPN solutions to be $£ 20000-£ 25000$, and the infusion pump and disposable equipment a further $£ 3000-£ 5000 .^{6}$ This was contrasted with the cost of one year in hospital of around $£ 100000$. Costs for our patients based on a year of HPN are made up by parenteral nutrition solutions $£ 15000$, disposables $£ 3000$, and pump rental $£ 1500$. The home care company charge $15 \%$ of this total as a service charge covering two week stock holding, free loan of trolley, refrigerator and drip stand, help to arrange holidays, fast response to change in regimen, and quarterly financial reports. In our trust, the cost of one year paediatric inpatient treatment including parenteral nutrition approximates to $£ 105000$.

Despite the apparent considerable cost advantage of HPN, Bisset et al found that difficulty agreeing funding arrangements in the absence of a universally accepted system often resulted in delay of discharge from hospital. In fact, both budget holders for community and hospital care may argue that they do not have resources allocated for long term home care treatment. Intravenous nutrient solutions can be prescribed by the general practitioner (following advice from the nutritional care team) using a form FP10; the cost is not included in the usual monitoring of practice drug expenditure. When seeking funding for the costs of other supplies and the services of the home care company, we first approach the locality manager of the community nursing services for the area in which the child lives. If there is a negative response, our trust takes financial responsibility and costs are recovered through contracting agreements with purchasers.

\section{Liaison with primary health care team}

First contact is with the general practitioner. A full medical summary is provided at the time it is decided that HPN is going to be pursued. The nutrition nurse then visits the general practitioner surgery to explain the implications and discuss prescribing and funding with the primary health care team. The health visitor and district nurse are invited to the ward for a case discussion and explanation of HPN, and joint home visits arranged after discharge. We have found that where available, the active involvement of a paediatric community nurse facilitates discharge and provides excellent local support for parents.

\section{Venous access}

In hospital and at home venous access is maintained by a central venous catheter. Through gradually increasing the amount of time disconnected from the parenteral nutrition infusion while in hospital, the aim of HPN is to give solutions over 12 hours at night so that the child is mobile and free of the infusion pumps during the day. Central venous catheters are usually of the Broviac or Hickman type, although we have discharged one child with a totally implantable venous access device because of problems with recurrent (Broviac) catheter sepsis while in hospital. The catheter tip is placed just within the right atrium and Broviac/Hickman lines exit from a skin tunnel below the nipple. There is less experience of long term parenteral nutrition using totally implantable devices which must be accessed using a Huber needle. Catheters are filled with heparin solution during the day but may block, acutely with blood, or slowly with a gradual build up of a hard waxy deposit which seems to be a mixture of both fat emulsion and endogenous lipids. If the central venous catheter is to be saved, blockage of either type needs prompt action which may involve a urokinase lock or infusion, hydrochloric acid, ethanol, or passage of a guide wire. ${ }^{18}$

Parents are taught about the signs of catheter sepsis, and to bring the child to the ward for review. After clinical assessment, peripheral and central venous catheter blood samples are taken for quantitative bacterial culture,,$^{19}$ and alternative sites of primary 
Table 2 Monitoring during long term parenteral nutrition

Monthly

Full blood count; clotting studies

Sodium; potassium; calcium; magnesium; phosphate chloride; creatinine; urea; alkaline phosphatase; albumin aspartate transaminase; bilirubin (conjugated/ unconjugated); ferritin; copper; zinc; selenium cholesterol; triglycerides

Every six months

Aluminium; chromium; manganese; folate; vitamins A, E $\mathrm{D}, \mathrm{B} 1, \mathrm{~B} 2, \mathrm{~B} 6, \mathrm{~B} 12$

infection excluded. In suspected catheter sepsis we commence treatment with intravenous gentamicin and vancomycin via the catheter itself, while awaiting the result of blood culture. Every effort is made to salvage blocked, infected, or damaged lines in children requiring long term intravenous treatment. If the catheter has to be removed, we try and place a new catheter at the same time through the existing track.

The obsessional and expert care given by parents at home probably explains the impression of a reduced risk of catheter sepsis in HPN compared with hospital patients, a finding noted in adult patients. ${ }^{20}$ It is particularly difficult for parents to witness the sometimes 'less than expert' attention of hospital staff when a child is readmitted. In this event, we encourage parents to continue performing those procedures for which they normally take responsibility at home.

\section{Support}

Continuing practical and psychological support for parents/home carers is essential. Parents meet with the hospital social worker and are given information about sources of financial assistance such as the disability living allowance. Help with disposable nappies may also be available. The support group for Patients on Intravenous and Nasogastric Nutrition Therapy (PINNT) share helpful advice, produce a newsletter, and organise meetings for patients and their families. An introduction to another family who have experience of HPN can be invaluable.

\section{Monitoring and follow up}

Once discharged from hospital, regular outpatient follow up is arranged initially at monthly intervals. Parenteral nutrition is generally safe, and although many different metabolic complications are described, sudden unexpected and serious biochemical disturbance seems relatively rare in stable patients both in hospital ${ }^{21}$ and at home. ${ }^{6}$ Trace element and vitamin requirements are, however, uncertain and should be monitored. Blood can be taken from the central venous catheter by the nutrition nurse at the time of clinic visit. In addition to anthropometry, we routinely check a range of haematological and biochemical parameters (table 2).

Concerns have been expressed regarding the frequency of thromboembolic events in children on long term parenteral nutrition, and also the risk of granulomatous pulmonary arteritis. ${ }^{22}$ For this reason we advocate an electrocardiogram twice a year and annual echocardiography. The risks of cholestatic liver disease and gall stones are increased both in short bowel syndrome and long term parenteral nutrition and can be monitored by abdominal ultrasound every six months. A yearly developmental assessment has also been advocated in children on HPN.

\section{Conclusion}

HPN requires a remarkable commitment from parents who, in return, must be adequately supported. The practical difficulties remain considerable and, as the number of suitable children is likely to remain small, experience should be built up in a restricted number of HPN centres. We believe that major advantages of HPN over hospital care include an improved quality of life, the opportunity for normal psychosocial development, decreased risk of catheter sepsis, and cost savings to the health service. It seems appropriate to paraphrase a much used aphorism in relation to choice of route for nutritional support, 'if the home works, use it'.

\section{Appendix}

We have used two of the home care companies: Fastnet - Fresenius Health Care Group, 6/8 Christleton Court, Stuart Road, Manor Park, Runcorn, Cheshire WA7 1ST; and Caremark - Caremark Ltd, 4 Kingsland Grange, Woolston, Warrington, Cheshire WA1 4RW.

Half-PINNT is the children's section of PINNT - Patients on Intravenous and Nasogastric Nutrition Therapy, 258 Wennington Road, Rainham, Essex RM13 9UU.

I would like to acknowledge the role of Sister Gill Abel, paediatric nutritional nurse specialist, in establishing HPN for children at the Leeds General Infirmary, and her helpful comments on this manuscript. I am grateful to Professor DCA comments on this manuscript. I am grateful to Professor DCA Candy and Dr S P Devane for permission to quote the findings
of the BPSU survey into children on long term parenteral of the BPSt
nutrition.

1 Council on Scientific Affairs. Home care in the 1990s. FAMA 1990; 263: 1241-4

Tatman MA, Woodroffe C. Paediatric care in the UK. Arch Dis Child 1993; 69: 677-80.

3 Payne-James J. Home care in the 1990s. Br f Hosp Med 1991; 46: 355

4 Anonymous. Who cares about homecare? Lancet 1991; 338:

1303-4.
5 BAPEN. Progress report of the British Association of Parenteral and Enteral Nutrition sub-committee on home artificial nutritional support. London: BAPEN, 1993.

6 Bisset WM, Stapleford P, Long S, Chamberlain A, Sokel B, Milla PJ. Home parenteral nutrition in chronic intestinal failure. Arch Dis Child 1992; 67: 109-14.

7 O'Connor MJ, Ralston CW, Ament ME. Intellectual and perceptual-motor performance of children receiving properceptual-motor performance of children receiving pro81: 231-6.

8 Ricour C, Gorski AM, Goulet O, et al. Home parenteral nutrition in children: 8 years of experience with 112 patrition in children: 8 years of experienc

9 Hancock BJ, Wiseman NE. Lethal short bowel syndrome. $\Im$ Pediatr Surg 1990; 25: 1131-5.

10 Schroeder P, Goulet O, Lear PA. Small bowel transplantation: European experience. Lancet 1990; 336: 110-1.

1 Goulet OJ, Revillon Y, Jan D, et al. Neonatal short bowel syndrome. F Pediatr 1991; 119: 18-23.

12 Booth IW. Enteral nutrition as primary therapy in short bowel syndrome. Gut 1994; suppl 1: S69-72.

13 Puntis JWL, Holden CE. Home enteral nutrition in paediatric practice. $\mathrm{Br} \mathcal{F}$ Hosp Med 1991; 45: 104-7.

14 Puntis JWL, Holden CE, Smallman S, Finkel Y, George RH, Booth IW. Staff training: a key factor in reducing 
intravascular catheter sepsis. Arch Dis Child 1991; 66: 335-7.

15 Puntis JWL, Booth IW. The place of a nutritional care team in paediatric practice. Intensive Therapy and Clinical Monitoring 1990: 132-6.

16 Lennard-Jones JE, chair. $A$ positive approach to nutrition as treatment. Report of a working party on the role of enteral and parenteral feeding in hospital and at home. London: King's Fund, 1992. King's Fund, 1992

17 Holden CE. Home parenteral nutrition for your child. Yorkshire: Blackwell Masters, 1993.

18 Ball PA, Booth IW, Holden CE, Puntis JWL. Paediatric parenteral nutrition. Milton Keynes: Pharmacia Limited, 1994: 69-74.
19 Ruderman JW, Morgan MA, Klein AH. Quantitative blood cultures and the diagnosis of sepsis in infants with umbilical and Broviac catheters. $\mathcal{F}$ Pediatr 1988; 112: 748-51.

20 Rannem T, Ladefoged $\mathrm{K}$, Tvede $M$, Lorentzen JE, Jarnum S. Catheter-related septicaemia in patients receiving home parenteral nutrition. Scand $f$ Gastroenterol 1986; 21: 455-60.

21 Puntis JWL, Hall SK, Green A, Smith DE, Ball PA, Booth IW. Biochemical stability during parenteral nutrition in children. Clinical Nutrition 1993; 12: 153-9.

22 Puntis JWL, Wilkins KM, Ball PA, Rushton DI, Booth IW. Hazards of parenteral treatment: do particles count? Arch Dis Child 1992; 67: 1475-7. 